\title{
Alternativas económicas para la determinación de la exactitud altimétrica de MDE obtenidas mediante técnicas LiDAR
}

\author{
Pablo Roces-Coto*
}

Recibido el 12 de mayo de 2014; aceptado el 14 de julio de 2014

\begin{abstract}
Land surveying and geodesic observations evolve at the same pace as the technology they use. Nowadays, airborne LiDAR measurements have turned into one of the most popular measurement methods. In DEM created by airborne LiDAR data, the height accuracy depends on a several amount of factors. With the appropriate analysis of all error sources in LiDAR measurements, final product's accuracy could be estimated. The influence of terrain slope and land covers are taken into account in every height accuracy assessment project for DEM generated by LiDAR techniques. But even more important is the accuracy assessment control, which is made by comparing the height data obtained by LiDAR for some control points. These points could exist previously to the work or should be measured for the occasion by other techniques. The quality of the DEM accuracy assessment control depends on which control points are used. In this paper the pros and cons of using some points or others for the accuracy estimation of DEM generated by LiDAR techniques are studied. There are three points kinds studied: high accuracy control points measured by GNSS on the field, survey marks previously materialized and DEM generated by classic photogrammetric methodology. The last two are far from the usual procedure and they optimize the control expenses. In the calculation, interpolation methods are used, therefore, the influence in the DEM accuracy of one method or other is studied. Putting the errors into tests of normality shows that they do not have this distribution due to the presence of outliers, so the use of robust statistics for the representation of the accuracy is recommended. Even though the measurement of control points on the field grants the highest accuracy and is the most common control method, it is also the most expensive. The use of previously
\end{abstract}

* ETSi Topografía, Geodesia y Cartografía, Universidad de Madrid, Campus Sur de la UPM, 28031, Madrid, España, correo electrónico: p.roces@outlook.com 
materialized survey marks shows up as a proper alternative and way cheaper (even free).

Key words: LiDAR, digital elevation model, errors, accuracy, control points.

\section{Resumo}

O campo das medições topográficas e geodésicas evoluiu no mesmo ritmo das tecnologias que as cerca. Atualmente as medições LiDAR aerotransportadas se impuseram como um método popular de medição. A exatitude altimétrica dos Modelo Digital de Elevação (MDE) gerados por vôos fotogramétricos com sensores LiDAR vem condicionada por muitos fatores. Com a devida análise das fontes de erros das medições LiDAR, se poderá estimar a exatitude a ser obtida no produto final. Em todos os estudos de determinação de exatitude altimétrica do MDE por técnicas LiDAR se tem em conta a influência da inclinação do terreno e da cobertura vegetal. Mas, ainda mais importante, será o próprio controle da exatitude. Este, é levado a cabo comparando-se os dados altimétricos obtidos com LiDAR com outros pontos de controle, que podem ser preeexistentes ou podem ser adquiridos de forma específica. A qualidade do controle da exatitude do MDE resultante dependerá de que pontos de controle se utilizem. No presente documento se estudam as vantagens e inconvenientes do uso de um ponto ou outro ao se determinar a exatitude de um MDE gerado por técnicas LiDAR. Os três tipos de pontos de controle estudados são: pontos de controle de alta precisão medidos em campo através de técnicas de GNSS, vértices de referência materializados previamente no terreno e MDE gerado por fotogrametria clássica. Os dois últimos métodos se desviam do procedimento habitual e otimizam os custos de controle. Nos cálculos que inserem processos de interpolação, se estuda também a influência da qualidade dos MDE no uso de alguns métodos ou outros. Ao se realizar testes de normalidade aos erros se confirma que os mesmos não seguem esta distribuição pela presença de outliers, e por isso que também se recomenda o uso de valores estatísticos robustos para a representação da exatitude. Embora a medição de pontos de controle de campo seja um método mais extenso e exato, também resulta ser o mais caro de todos. $\mathrm{O}$ uso de vértices de referência preestabelecidos se mostra como uma alternativa adequada e muito mais econômica (inclusive gratuita).

Palavras chave: LiDAR, Modelo Digital de Elevação, erros, exatitude e pontos de controle.

\section{Resumen}

El campo de las mediciones topográficas y geodésicas evoluciona al mismo ritmo que la tecnología de la que se sirve. En la actualidad las mediciones LiDAR aerotransportadas se han impuesto como un método popular de medición. La exactitud 
altimétrica de los MDE generados por vuelos fotogramétricos con sensores LiDAR viene condicionada por muchos factores. Con el debido análisis de las fuentes de error de las mediciones LiDAR, se podrá estimar la exactitud que se obtendrá en el producto final. En todos los estudios de determinación de exactitud altimétrica de MDE por técnicas LiDAR se tienen en cuenta la influencia de la pendiente y las cubiertas vegetales. Pero aún más importante será el propio control de la exactitud. Éste es llevado a cabo comparando los datos altimétricos obtenidos por el LiDAR con otros puntos de control, que pueden ser preexistentes o pueden ser adquiridos específicamente. La calidad del control de la exactitud del MDE resultado dependerá de qué puntos de control se utilicen. En el presente documento se estudian las ventajas e inconvenientes del uso de unos puntos $\mathrm{u}$ otros a la hora de la determinación de la exactitud de un MDE generado por técnicas LiDAR. Los tres tipos de puntos de control estudiados son: puntos de control de alta precisión medidos en campos mediante técnicas GNSS, vértices de referencia materializados previamente en el terreno y MDE generados por fotogrametría clásica. Los dos últimos métodos se alejan del procedimiento habitual y optimizan costes del control. En los cálculos intervienen procesos de interpolación, por lo que se estudia también la influencia en la calidad del MDE del uso de unos métodos frente a otros. Al realizarse los test de normalidad a los errores se confirma que los mismos no siguen esta distribución por la presencia de outliers, por lo que también se recomienda el uso de valores estadísticos robustos para la representación de la exactitud. Pese a que la medición de puntos de control en campo es el método más extendido y exacto, también resulta ser el más caro de todos. El uso de vértices de referencia previos se muestra como una alternativa adecuada y mucho más económica (incluso gratuita).

Palabras clave: LiDAR, Modelo Digital de Elevación, errores, exactitud, puntos de control.

\section{Introducción}

El acrónimo LiDAR proviene del inglés Light Detection and Ranging. Este tipo de sensores requiere la emisión y detección en su camino de vuelta de millones de pulsos de luz o láser y la medición indirecta de la distancia recorrida por los mismos a través del tiempo de viaje requerido. El gran número de pulsos láser emitidos permiten atravesar las cubiertas de vegetación entre espacios muy pequeños de ésta para una misma posición espacial plana, por lo que se pueden obtener al mismo tiempo puntos a diferentes alturas como, por ejemplo, en la copa de un árbol y en el suelo. En la actualidad, los sensores LiDAR están sustituyendo progresivamente a los sensores fotogramétricos más clásicos en la obtención de Modelos Digitales de Elevación (MDE), pese a que los métodos clásicos siguen ofreciendo resultados adecuados y hasta no hace demasiado tiempo, más económicos (Dehvari y Heck, 2012). La generación de MDE es hoy en día la aplicación principal de los vuelos 
con sensores LiDAR (Liu, 2011) y para determinar la exactitud de este tipo de productos es necesario llevar a cabo unas tareas de control con datos de campo. El proceso más habitual es la toma de una serie de puntos de control en el campo, los cuales han de ser medidos con instrumental de alta exactitud. A pesar de que este método resulta ser el que asegura unos resultados más exactos (Hodgson y Bresnahan, 2004) y en el que más se ha trabajado para minimizar sus errores (Aguilar et al., 2009) (Höhle y Höhle, 2009), también se trata de un proceso de altísimo coste por el tiempo que requiere, el instrumental utilizado y los operarios especializados necesarios. En el presente documento se desean introducir métodos alternativos que se basan en la reutilización de información ya disponible y que minimizan los costes del control de exactitud. Los tipos de datos alternativos que se analizarán serán: vértices de referencia previos al trabajo, como puedan ser estaciones de control para mediciones GNSS o vértices pertenecientes a redes geodésicas (Liu, 2011) y MDE obtenidos por fotogrametría clásica (Dehvari y Heck, 2012), de los que suele haber acceso sencillo y a bajo coste. Los estándares presuponen que el método de control utilizado deberá ser tres veces más exacto que el dato que se quiere controlar (NDEP, 2004), por lo que comparar un MDE LiDAR con un MDE fotogramétrico tendrá sentido siempre que se cumpla esta condición. Usualmente estos últimos sólo describen la superficie del terreno. Si se estima el error del MDE LiDAR por este procedimiento, este dato se puede extrapolar a otros MDT generados por el sensor LiDAR, como puede ser un MDS de las copas de los árboles. Por otro lado, también se analizarán las ventajas e inconvenientes del uso de puntos de control medidos expresamente en campo para el trabajo, al que se le trata de encontrar alternativas.

Independientemente del método de control utilizado, existen factores que influyen en la calidad del MDE y que deben al menos reseñarse. El primero será las condiciones del terreno: la pendiente hará variar de manera importante la exactitud altimétrica que se puede lograr en el MDE (Dehvari y Heck, 2012) (Höhle y Höhle, 2009) y también el tipo de cubiertas del terreno (Liu, 2011) (Hladik y Alber, 2012) (Hodgson y Bresnahan, 2004) (Höhle y Höhle, 2009). El segundo serán las herramientas de cálculo utilizadas en la determinación de la exactitud: como diferentes métodos de interpolación de cotas (Liu, 2011) y el uso o no de métodos estadísticos robustos para la estimación de la exactitud que consideren la presencia de outliers en las estimaciones del error (Höhle y Höhle, 2009).

Para estimar las exactitudes altimétricas de los vuelos LiDAR que se pueden llegar a alcanzar se han realizado trabajos y proyectos que la delimitaron entre los $10-50 \mathrm{~cm}$ de error medio cuadrático (RMSE, Root Mean Square Error) a $68 \%$ de nivel de confianza o $19.6-98 \mathrm{~cm}$ a $95 \%$ de nivel de confianza [Inter-Governmental Committee on Surveying and Mapping (ICSM, 2008)]. Se podrán obtener exactitudes superiores (10-15cm de RMSE a $68 \%$ de nivel de confianza) en condiciones ideales, tanto de pendiente como de cubierta del terreno (Hodgson y Bresnahan, 
2004). Estas exactitudes dependerán de las características del vuelo (i.e. altura de vuelo, velocidad de pasada, longitud de onda, etcétera), pero en todo caso para su determinación será necesaria la comparación de las alturas estimadas por el LiDAR con el dato de campo en los denominados puntos de control o checkpoints. Estos puntos serán independientes del proceso de producción del MDE, y únicamente se utilizarán para la determinación su exactitud. Normalmente, la tarea de toma de puntos de control (si es necesaria) será llevada a cabo por una empresa independiente al vuelo [National Digital Elevation Program (NDEP, 2004)]. Se recomienda que los puntos de control se encuentren distribuidos por todos los diferentes tipos de cubiertas vegetales de la zona de estudio (NDEP, 2004) en un volumen de al menos 20 puntos por tipo de cubierta [American Society for Photogrammetry and Remote Sensing (ASPRS, 2004)]. La toma de tal volumen de información a los niveles de exactitud exigidos es una tarea que supone altos costes y requiere tiempo. El uso de métodos alternativos de menor coste en la determinación de la exactitud del MDE busca mejorar la relación calidad-precio de éste.

Los vértices ya materializados en el terreno pertenecientes a redes de control nacional, estaciones de control de mediciones GNSS u otros similares, cualquiera que sea su utilidad, son típicamente un dato altimétrico de alta exactitud, a cuya información se puede acceder de manera gratuita habitualmente. Por otro lado, los MDE generados por métodos de fotogrametría clásica suelen formar parte de las bases de datos nacionales y existen planes que cubren todo el territorio nacional, como el Plan Nacional de Ortofotografía Aérea (PNOA) para España, que también incluye cobertura total por ortofotografías y LiDAR en la mayoría del territorio, pudiéndose acceder a la información a un bajo coste.

Tanto en el uso de puntos de control habituales como en los vértices de redes, los haces LiDAR no coincidirán nunca con la posición planimétrica de éstos. Por ello, será necesario el uso de métodos de interpolación de las cotas estimadas por LiDAR en la ubicación de los puntos en el terreno. El uso de un método de interpolación u otro puede ayudar a determinar la calidad del MDE con mayor o menor exactitud (Liu, 2011), ya que cada uno de los métodos de interpolación devolverá valores de la cota diferentes. Siempre es posible eliminar los errores propios de llevar a cabo una interpolación mediante el replanteo de los puntos de control en el campo en las posiciones exactas tomadas por el sensor LiDAR (Hodgson y Bresnahan, 2004), pero ello no es habitual.

Las herramientas estadísticas utilizadas para representar la exactitud también tendrán un papel en un estudio de este tipo. La presencia de outliers en los valores de los errores implicará que la distribución de éstos no sea normal (Höhle y Höhle, 2009). Para estimar la exactitud será necesario, por tanto, el uso de herramientas estadísticas robustas, ya que valores como el error medio cuadrático (RMSE) son muy afectados por los outliers que empobrecerían los resultados. El uso o no de 
estas herramientas será analizado y se verá de qué manera afecta a la calidad de la determinación de la exactitud.

El objetivo del documento será mostrar las ventajas del uso de fuentes alternativas de puntos de control a utilizar en la determinación de la exactitud de los MDE, al mismo tiempo que se reseñan herramientas de cálculo que permiten expresar la exactitud más adecuadamente y más cercana a la realidad.

\section{Condiciones del terreno}

Sea cual sea el dato de control de la exactitud del MDE, éste siempre se verá afectado por las condiciones del terreno y el usuario no lo podrá evitar de ningún modo. Por este motivo, el usuario debe estar prevenido del efecto de la naturaleza de la superficie en la exactitud de los MDE generados por técnicas LiDAR. Es conocida la influencia de las cubiertas del suelo en la capacidad de rebote de los haces láser, pero la pendiente del terreno puede inducir a problemas altimétricos de mayor importancia (Hodgson y Bresnahan, 2004).

A continuación, se detallan ambos factores para poder conocer la influencia de éstos en la exactitud del MDE.

\section{Pendiente}

Se conoce y se ha documentado desde hace mucho tiempo la importancia de la pendiente en los errores altimétricos para la elaboración de cualquier tipo de mapa, independientemente del método de creación utilizado (Mailing, 1989). Estos mismos errores se presentan en las mediciones LiDAR, y es que aunque una cota se pudiese llegar a medir sin ningún error, los errores planimétricos introducirían errores aparentes, únicamente presentes en los terrenos con una cierta inclinación.

El error será máximo cuando el desplazamiento planimétrico ocurra en dirección de la línea de máxima pendiente, y cero si se produce en dirección perpendicular (Hodgson y Bresnahan, 2004). Resulta imposible conocer la dirección de desplazamiento de un punto concreto, por lo que se asume que ésta es aleatoria en toda la nube de puntos.

Para hallar un error medio cuadrático en altimetría debido a la pendiente, que se denominará $\mathrm{RMSE}_{\text {pendiente, }}$ se asume que tanto el desplazamiento horizontal como la pendiente tienen una distribución gaussiana, con una pendiente media que oscila entre $\operatorname{los} 0^{\circ}$ y $40^{\circ}$, con una desviación típica $\left(\sigma_{\text {pendiente }}\right)$ entre $0.5^{\circ}$ y $1^{\circ}$ (Hodgson y Bresnahan, 2004). Con una muestra aleatoria de 17 puntos, cuyas cotas se midieron en campo mediante el instrumental adecuado, se obtuvo la siguiente fórmula (Hodgson y Bresnahan, 2004): 


$$
\begin{aligned}
R M S E_{\text {pendiente }}= & \left(0.6893 * \tan (\overline{\text { pendiente }}) * R M S E_{\text {horizontal }}\right) \\
& +\left(0.0062 * R M S E_{\text {horizontal }}+\sigma_{\text {pendiente }}\right)
\end{aligned}
$$

La pendiente media se calculará como la media aritmética de todas las pendientes estimadas a partir de la red irregular de triángulos (TIN) formada por los puntos de la muestra. Al mismo tiempo, se hallará la desviación estándar ( $\left.\sigma_{\text {pendiente }}\right)$. El RMSE $_{\text {horizontal }}$ se refiere a la exactitud planimétrica o desplazamiento horizontal del sensor de captura del vuelo, en este caso, el sensor LiDAR.

\section{Tipo de cubierta}

Dependiendo de la zona de estudio podrán existir muchos tipos de cubiertas en las que analizar los resultados de exactitud (Liu, 2011) (Hodgson y Bresnahan, 2004). Los pulsos láser emitidos por el sensor LiDAR son tan numerosos que pueden obtener información a través de ciertos tipos de cubierta para una misma posición plana, por lo que una de las ventajas en este tipo de mediciones es que su registro no se limitará a las capas superiores. Un pulso que llega a una zona boscosa podrá tener diferentes retornos: uno en la copa del árbol, otro en una altura intermedia y finalmente, en el suelo. Ésta sería una situación ideal, ya que resulta común que si la vegetación es demasiado densa, no existirán huecos que permitan la llegada del pulso al suelo. A la hora de realizar un MDE es el dato del suelo el que se quiere registrar, por lo que en zonas de vegetación densa la exactitud será menor, ya que los haces no llegan al suelo. Se tiene que estimar la cota a partir de los puntos más cercanos, por lo que la exactitud será menor (Aguilar et al., 2009).

En el trabajo señalado se consideran seis tipos de cubiertas a estudiar: pavimento o urbano, hierba baja (hasta $\operatorname{los} 8 \mathrm{~cm}$ ), hierba alta (hasta $10 \mathrm{c} 90 \mathrm{~cm}$ ), matorrales y árboles bajos, bosque perenne (principalmente pinares) y bosque caduco. En la Tabla 1 pueden consultarse los resultados del RMSE para los diferentes tipos de cubiertas, en función también de su pendiente media y al mismo tiempo de una pendiente supuesta de $25^{\circ}$, con $5^{\circ}$ de desviación estándar. Los puntos de control fueron medidos en campo de forma bastante inusual: mediante replanteo en el terreno de algunas de las coordenadas obtenidas por sensor LiDAR. Teóricamente al realizar este proceso, los puntos de control y los puntos LiDAR se encontrarán en la misma posición, por lo que las cotas de éstos podrán ser comparadas directamente sin la necesidad de llevar a cabo una interpolación. Las fórmulas a partir de las cuales se calculan los errores medios cuadráticos son las siguientes (Hodgson y Bresnahan, 2004): 


$$
R M S E_{\text {Pts LiDAR observados }}=\sqrt{\frac{\sum\left(Z_{\text {LiDAR }}-Z_{\text {campo }}\right)^{2}}{n}}
$$

$$
R M S E_{\text {LiDAR }}=\sqrt{R M S E_{\text {Pts LiDAR observados }}^{2}-R M S E_{\text {medida }}^{2}-R M S E_{\text {pendiente }}^{2}}
$$

El RMSE $E_{\text {medida }}$ se refiere al error medio cuadrático de las propias mediciones láser, tanto errores de emisión como en el posicionamiento de los fotocentros.

Tabla 1

Errores medios cuadráticos en metros debidos a las condiciones del terreno

\begin{tabular}{lcccccc}
\hline & \multicolumn{7}{c}{ Tipo de cobertura } \\
\cline { 2 - 7 } & \multicolumn{7}{c}{ Pavimento } & Hierba baja & Hierba alta & Matorral & Perenne & Caduco \\
\hline Pendiente media & $1.67^{\circ}$ & $2.27^{\circ}$ & $1.08^{\circ}$ & $4.15^{\circ}$ & $2.30^{\circ}$ & $2.50^{\circ}$ \\
RMSE Pts LiDAR & 0.189 & 0.225 & 0.189 & 0.233 & 0.172 & 0.259 \\
RMSE LiDAR & 0.184 & 0.214 & 0.185 & 0.213 & 0.163 & 0.252 \\
RMSE Pts LiDAR & & & & & & \\
$\left(25^{\circ}\right.$ de pendiente) & 0.462 & 0.475 & 0.463 & 0.474 & 0.454 & 0.493 \\
\hline
\end{tabular}

Fuente: Hodgson y Bresnahan, 2004.

Analizando los resultados, los autores señalan que sorprende que los errores sean menores en zonas boscosas de hoja perenne que en hierba baja, por ejemplo. Esto parece ser debido a tratarse de zonas de pinares, cuyas hojas tienen muy poca cantidad de agua y permiten un paso muy limpio de los haces láser, además de facilitar el retorno en el suelo por la capa de hojas caídas siempre presente en este tipo de bosques. Por lo demás, los resultados se encuentran dentro de los valores esperados. En la Tabla 1 queda presente la importancia de conocer los valores de pendiente, ya que en pendientes altas los valores de los errores aumentan muy visiblemente, e incluso consiguen que las exactitudes sean más bajas en las cubiertas de hierba baja que la hierba alta.

Conocer el tipo de cubiertas de la zona de estudio y disponer de una clasificación previa de éstas puede mejorar la exactitud de un MDE con respecto a este factor (Hladik y Alber, 2012).

\section{Control de exactitud}

Con el fin de verificar la calidad y exactitud final del MDE generado por el vuelo LiDAR será necesario contrastar los valores obtenidos con otros datos. Este proceso puede acarrear un gran volumen de trabajo en un proyecto de este tipo, e incluso 
aumentar los gastos considerablemente. Por ello, se busca la reutilización de información destinada a otros objetivos en estas tareas, que puedan ayudar a optimizar costes y esfuerzos. Se ha de ser consciente de que los datos que se utilicen en la comparación aunque sean más exactos no se encontrarán exentos de error, ya que resulta imposible conocer la "verdad" del terreno.

En este apartado se mostrará la viabilidad de diferentes modos para comprobar la exactitud de un MDE generado por un vuelo LiDAR comenzando por lo métodos alternativos, más económicos: compararlo con un MDE obtenido por fotogrametría convencional (Dehvari y Heck, 2012) y con vértices ya materializados en el terreno independientemente del vuelo (Liu, 2011). Al mismo tiempo que se estudia si los vértices resultan adecuados en este tipo de tareas, se comprueba la influencia en la exactitud de diferentes tipos de interpolación de cotas.

A continuación, se expondrán las ventajas e inconvenientes del uso de puntos de control. Este método es el más extendido y del que se trata de buscar alternativas por los altísimos costes que supone. En este último apartado también se estudia la influencia del uso de herramientas estadísticas adecuadas para la representación de la exactitud en presencia de outliers.

\section{MDE obtenidos por otros medios}

En la actualidad los costes de producción de un MDE por técnicas LiDAR son muy similares a los de un MDE a partir de un vuelo fotogramétrico convencional, pero no era el caso hace poco tiempo. Comparar las exactitudes entre estos dos tipos de MDE permitirá saber si un MDE fotogramétrico puede ser utilizado como dato de control en la determinación de la exactitud de un MDE LiDAR. La mayoría de países desarrollados cuentan con planes para la elaboración de ortofotografías nacionales, así como de MDE, por lo que el acceso a estos datos resulta sencillo y barato, e incluso gratuito.

La elaboración de MDE por técnicas de fotogrametría clásica se basa en el cálculo de las funciones de colinealidad mediante el uso de puntos de control en el suelo de coordenadas conocidas (medidos en campo con GNSS diferencial) que se identificarán como puntos homólogos en pares estereoscópicos de imágenes. En el trabajo de referencia se comparó este tipo de MDE con el generado por LiDAR en una zona rural de Huron, Ontario, Canadá (Dehvari y Heck, 2012).

A partir de aproximadamente 120 puntos de revisión independientes a ambos MDE se calcularon los valores de exactitud básicos como los RMSE, tanto altimétricos como planimétricos, y otros valores estadísticos de los MDE en función de las pendientes y sus direcciones. Los resultados se ven reflejados en la Tabla 2, en donde el primer grupo de valores se corresponden a altimetría y el segundo a planimetría en direcciones Este-Oeste (x) y Sur-Norte (y). RMSE será el error medio cuadrático; $S$, la desviación estándar del error total; y SRMSE, la desviación 
estándar del RMSE. Con los resultados se puede observar que en cuestión de exactitud altimétrica, los métodos LiDAR son superiores; mientras que al mismo tiempo la fotogrametría clásica asegura unas exactitudes planimétricas muy superiores.

Tomar los datos del MDE de fotogrametría clásica como veraces y compararlos con los del generado por técnicas LiDAR implicaría asumir unos errores en torno a los $10 \mathrm{~cm}$ de incertidumbre a sumar a la exactitud final del MDE. Sabiendo que el punto de control utilizado debe ser tres veces más exacto que el dato que se quiere controlar (NDEP, 2004), los MDE generados por métodos fotogramétricos no servirán para llevar a cabo tareas de control por no alcanzar dicha exactitud altimétrica, aunque sí serían válidos para la determinación de la exactitud planimétrica.

Tabla 2

Comparación de exactitudes de los MDE generados a partir de LiDAR y fotogrametría clásica. Unidades en metros

\begin{tabular}{lcc}
\hline \multicolumn{1}{c}{ Estadístico } & LiDAR & $\begin{array}{c}\text { Fotogrametría } \\
\text { clásica }\end{array}$ \\
\hline$R M S E_{v}$ & 0.20 & 0.29 \\
$S_{v}$ & 0.20 & 0.30 \\
$S_{R M S E v}$ & 0.03 & 0.20 \\
Error absoluto vertical & 0.14 & 0.20 \\
$R M S E_{x}$ & 0.37 & 0.01 \\
$R M S E_{y}$ & 0.48 & 0.04 \\
$S_{x}$ & 0.36 & 0.02 \\
$S_{R M S E x}$ & 0.06 & 0.00 \\
Error absoluto horizontal (x) & 0.27 & 0.01 \\
$S_{y}$ & 0.48 & 0.04 \\
$S_{R M S E y}$ & 0.07 & 0.01 \\
Error absoluto horizontal (y) & 0.45 & 0.02 \\
\hline
\end{tabular}

Fuente: Dehvari y Heck, 2012.

\section{Vértices de redes previas}

La toma de puntos de control y verificación en campo supone un sobrecoste para cualquier tipo de trabajo fotogramétrico ya que requerirá disponer de más operarios especializados, múltiples jornadas de trabajo y el uso de equipamiento de alta precisión si se quiere asegurar resultados óptimos. Una alternativa a este inconveniente que se plantea es la utilización para dichas tareas de vértices materializados en el terreno con anterioridad al proyecto de generación del MDE (Liu, 2011). Para ello, se pueden utilizar estaciones de referencia que se encuentren en continua medición de sus coordenadas mediante posicionamiento absoluto por métodos GNSS. Existen países en los que no hay una gran disponibilidad de estos puntos, por lo que una alternativa a éstos son vértices pertenecientes a redes geodésicas de cualquier orden presentes en cualquier país con una cartografía regulada. La principal ventaja 
de estos puntos es que se puede disponer de su altura de manera gratuita, ya que disponen de reseñas que suelen ser accesibles por internet. Por el contrario, la exactitud con la que se determinará el error del MDE será menor, ya que el volumen de puntos de control a utilizar será muchísimo más bajo que en un proceso habitual de medición de puntos de control en campo. Por ejemplo, la Red de Orden Inferior (ROI) de España se trata de una red de vértices geodésicos de $7 \mathrm{~km}$ de lado, lo que supone una densidad de un vértice cada $45 \mathrm{~km}^{2}$. También podría servir de ejemplo la Red de Nivelación de Alta Precisión (REDNAP), la cual cuenta con puntos materializados en carreteras, principalmente, con alta exactitud altimétrica y distanciados entre sí del orden de $1 \mathrm{~km}$. Existen redes de este tipo en multitud de países, como por ejemplo la Deutsches Hauptdreiecksnetz (DNDN) de Alemania, o todas las redes de la National Geodetic Survey (NGS) de los Estados Unidos de América.

La probabilidad de que dichos vértices, al igual que sucedería con puntos de control medidos para el proyecto, coincidan con los rebotes de los haces láser es muy baja, por lo que será necesario el uso de métodos de interpolación para estimar la altura en la ubicación del vértice. Dependiendo del método de interpolación utilizado los resultados también variarán, por lo que se probaron cinco métodos diferentes. También existe una última prueba a realizar, si se quiere hallar la exactitud del MDE a 95\% de confianza, se deberá saber si la distribución de los errores es gaussiana. En caso de serlo, la exactitud podrá expresarse como 1.96 x RMSE (ASPRS, 2004); pero si la distribución no es normal (problema normalmente ocasionado por la presencia de outliers) deberán utilizarse métodos estadísticos robustos, como el 95 percentil (ASPRS, 2004) (Höhle y Höhle, 2009). Para comprobar la distribución de los errores se calcularon histogramas de frecuencia y gráficos cuantil-cuantil (QQ) para múltiples tipos de cubiertas.

En la Figura 1 puede comprobarse que los errores no siguen una distribución normal. El motivo por el que no se cumple esta distribución es por la presencia de outliers (Liu, 2011), por lo que el modo más adecuado de representar la exactitud será mediante métodos estadísticos robustos. En la Tabla 3 se pueden ver los resultados finales obtenidos para cinco diferentes tipos de cubiertas y cinco tipos de interpolación: inverse distance weighting (IDW), Kriging, polinomio local (LP), redes de triángulos (TIN) y, por último, el vecino más próximo (NP).

En los resultados de la Tabla 3 se aprecia cómo los métodos estadísticos robustos ofrecen exactitudes más altas y también que el método de interpolación más exacto es el de Kriging. En todo caso, los errores rondan $10 \mathrm{~s} 50 \mathrm{~cm}$.

Los valores de exactitud mediante el uso de vértices materializados previamente se encuentran dentro de los límites esperados de 19.6-98cm a 98\% de confianza (ICSM, 2008), por lo que se muestra como un método ideal en la determinación de la exactitud altimétrica de un MDE generado por técnicas LiDAR, tanto por exactitud como por coste. 

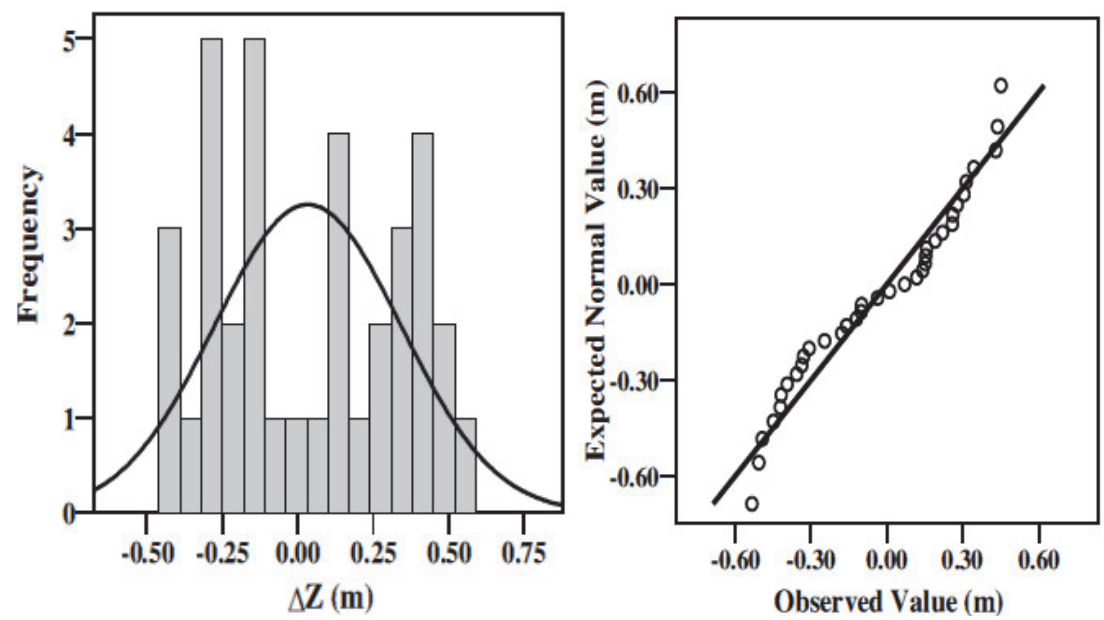

Figura 1. Comprobación de la distribución de los errores altimétricos en una cubierta de hierba para mediciones LiDAR. A la izquierda, histograma de frecuencias. A la derecha, gráfico Q-Q (Liu, 2011).

Tabla 3

Exactitud altimétrica en función del método de interpolado. Unidades en metros

\begin{tabular}{lllllll}
\hline Cubierta & Estadistico & IDW & Kriging & LP & TIN & NP \\
\hline Pavimento & 1.96* RMSE & 0.53 & 0.49 & 0.51 & 0.53 & 0.57 \\
& 95 Percentil & 0.50 & 0.44 & 0.47 & 0.52 & 0.56 \\
Hierbas & 1.96* RMSE & 0.61 & 0.61 & 0.57 & 0.65 & 0.74 \\
& 95 Percentil & 0.54 & 0.50 & 0.49 & 0.53 & 0.64 \\
Matorrales & 1.96* RMSE & 0.47 & 0.49 & 0.45 & 0.51 & 0.61 \\
& 95 Percentil & 0.41 & 0.41 & 0.38 & 0.41 & 0.53 \\
Arboledas & 1.96* RMSE & 0.49 & 0.49 & 0.49 & 0.51 & 0.59 \\
& 95 Percentil & 0.44 & 0.48 & 0.45 & 0.45 & 0.51 \\
Combinados & $1.96 *$ RMSE & 0.53 & 0.51 & 0.51 & 0.55 & 0.63 \\
& 95 Percentil & 0.50 & 0.48 & 0.47 & 0.51 & 0.57 \\
\hline
\end{tabular}

Fuente: Liu, 2011.

\section{Puntos de control}

La medida en campo de puntos de control será la solución más habitual en la determinación de exactitud de un MDE, sea cual sea su naturaleza. Ya se ha comentado en apartados anteriores la necesidad de que no sólo las medidas LiDAR sean precisas, sino que además tendrán que serlo las medidas de campo y también los métodos de cálculo y estadísticos de los errores. Para ilustrar este apartado se utilizarán los datos de un trabajo en el que se comprobó la necesidad del uso de méto- 
dos estadísticos robustos para el cálculo de la exactitud de un MDE en la zona de Aalborg, Dinamarca (Höhle y Höhle, 2009). Los puntos de control fueron medidos en campo con una distribución aleatoria y mediante métodos GNSS/RTK diferenciales, referenciados a vértices de redes superiores. El volumen de datos medidos en campo es enorme con un total de 126,559 puntos de revisión y a la hora del cálculo de la exactitud del MDE se decidió utilizar para la interpolación de las cotas de los puntos de retorno el método bilineal. De esta nube de puntos, sólo 19 son de alta precisión, de los cuales se derivan el resto. Aún así, suponen una gran inversión económica y de tiempo.

Como ya se señaló, la distribución de los errores altimétricos de las mediciones LiDAR no es normal, por lo que el uso de métodos estadísticos convencionales para la determinación de la exactitud arrojará peores resultados. Un método para la utilización de valores estadísticos convencionales es la detección y eliminación de outliers imponiendo un límite a partir del cual un valor se elimina. En este caso concreto, se eliminaron los valores que se alejaban de la media tres veces el valor del RMSE o más.

Los métodos estadísticos robustos utilizados para la determinación de la exactitud serán los cuantiles $(50 \%, 68.3 \%$ y 95\%), los cuales se corresponderían con los valores estadísticos de mediana, desviación estándar y valores del RMSE a 95\% de confianza en una distribución normal. En cuanto a la media, será calculada como la mediana normalizada absoluta (NMAD), la cual se calcula del siguiente modo:

$$
N M A D=1.4826 \cdot \text { mediana }\left(\left|\Delta h-m_{\Delta h}\right|\right)
$$

En donde la mediana se corresponderá con el valor de $50 \%$ cuantil y $m_{\Delta h}$ será la media de los errores.

Tabla 4

Exactitud altimétrica del MDE con puntos de control en función de los métodos estadísticos utilizados para el cálculo. Unidades en metros

\begin{tabular}{llllc}
\hline Distribución normal & \multicolumn{4}{c}{ Distribución no normal } \\
\hline \multicolumn{1}{c}{ Estadistico } & Valor & Estadistico & Valor & Intervalo (95\% conf.) \\
\hline RMSE & 1.06 & $50 \%$ cuantil & 0.02 & {$[0.01,0.02]$} \\
Media $(\mu)$ & 0.13 & NMAD & 0.12 & {$[0.12,0.13]$} \\
Desviación estándar $(\sigma)$ & 1.05 & $68.3 \%$ cuantil & 0.13 & {$[0.13,0.14]$} \\
$\mu$ (sin outliers) & 0.02 & $95 \%$ cuantil & 0.68 & {$[0.62,0.88]$} \\
$\sigma$ (sin outliers) & 0.34 & & & \\
\hline
\end{tabular}

Fuente: Höhle y Höhle, 2009. 
En la Tabla 4 puede comprobarse que, como se esperaba, los valores estadísticos robustos ofrecen unas exactitudes en la estimación del error más altas. También se puede ver que el uso de puntos de control frente al de vértices previos asegura una estimación del error del MDE más exacta, pero con una mayor inversión económica, de tiempo y de cálculo.

\section{Conclusiones}

El uso de unas herramientas u otras en la determinación de la exactitud de un MDE generado por técnicas LiDAR, tanto físicas como de cálculo, implica llevar a cabo el estudio de la relación calidad-precio. Tomando este referente, el uso de vértices de redes fijas previas a la medición con el sensor LiDAR se muestra como la mejor solución de todas. El que en principio se presentaba como un método alternativo, resulta ser una solución idónea, ya que ofrece unos resultados de exactitud dentro de los esperados en un trabajo de este tipo con un coste bajo y con una optimización de los procesos de cálculo, ya que el volumen de datos utilizado es mucho menor.

Por otro lado, los MDE generados mediante fotogrametría clásica no alcanzan la exactitud necesaria como para ser utilizados en la determinación de la exactitud de un MDE generado por LiDAR. También se pueden observar los buenos resultados del uso de puntos de control medidos en campo a la hora de determinar la exactitud altimétrica. Estos resultados sólo se producen en condiciones óptimas y mediante el uso de un gran volumen de datos que en muchos casos no merecerán su inversión económica.

A lo largo de todo este estado del arte también se ha querido remarcar la necesidad de conocer los métodos de cálculo más indicados en este tipo de operaciones, entre los cuales el Kriging se muestra como el mejor método de interpolación, y que debido a la distribución no normal de los errores altimétricos por la presencia de outliers, la exactitud quedará mejor representada mediante métodos estadísticos robustos.

\section{Bibliografía}

Aguilar, F.J.; Mills, J.P.; Delgado, J.; Aguilar, M.A.; Negreiros, J.G. y J.L. Perez (2009). "Modelling Vertical Error in LiDAR-Derived Digital Elevation Models", ISPRS Journal of Photogrammetry and Remote Sensing, núm. 65, pp. 103-110.

American Society for Photogrammetry and Remote Sensing (ASPRS) (2004). "Guidelines, Vertical Accuracy Reporting for LiDAR Data", <http://cort.as/4jQy>, visto el 6 de julio de 2013.

Dehvari, A. y Heck R.J. (2012). "Removing Non-Ground Points from Automated Photo-Based DEM and Evaluation of its Accuracy with LiDAR DEM", Computers \& Geosciences, núm. 43, pp. 108-117. 
Hladik, C. y M. Alber (2012). "Accuracy Assessment and Correction of a LiDARDerived Salt Marsh Digital Elevation Model", Remote Sensing of Environment, núm. 121, pp. 225-235.

Hodgson, M.E. y Bresnahan, P. (2004). "Accuracy of Airborne LiDAR-Derived Elevation: Empirical Assessment and Error Budget", Photogrammetry Engineering and Remote Sensing, núm. 70, pp. 331-339.

Höhle, J. y Höhle, M. (2009). "Accuracy Assessment of Digital Elevation Models by Means of Robust Statistical Methods", ISPRS Journal of Photogrammetry and Remote Sensing, núm. 64, pp. 398-406.

Inter-Governmental Committee on Surveying and Mapping (ICSM) (2008). "Guidelines for Digital Elevation Data", $<$ http://cort.as/4jQx $>$, visto el 6 de julio de 2013.

Liu, X. (2011). "Accuracy Assessment of LiDAR Elevation Data Using Survey Marks", Survey Review, núm. 43, pp. 80-93.

Mailing, D.H. (1989). "Measures from Maps", Pergamon Press, New York.

National Digital Elevation Program (NDEP) (2004). "Guidelines for Digital Elevation Data". Version 1.0. <http://cort.as/4jR->, visto el 15 de julio de 2013. 